\title{
The Inverse Amplitude Method and Heavy Baryon Chiral Perturbation Theory applied to pion-nucleon scattering *
}

\author{
J. R. Peláez ${ }^{a}$ and A. Gómez Nicola ${ }^{a}$, \\ a Departamento de Física Teórica. Universidad Complutense. 28040 Madrid. SPAIN.
}

We report on our present work, where by means of the Inverse Amplitude Method we unitarize the elastic pion nucleon scattering amplitudes of Heavy Barion Chiral Perturbation Theory at $O\left(q^{3}\right)$. We reproduce the scattering up to the inelastic thresholds including the $\Delta(1232)$ resonance. The fitted chiral constants are rather different from those obtained by fitting the extrapolated threshold parameters for the non-unitarized theory.

\section{Introduction: Heavy Baryon Chiral Perturbation Theory}

Chiral Symmetry is a relevant constraint in the interactions between pions and nucleons, as it is well known since current algebra appeared in the sixties. However, in order to go beyond the current algebra results or tree level calculations from simple models, one needs an effective theory with a systematic power counting. Heavy Barion Chiral Perturbation Theory (HBChPT) [1] is an Effective Theory of nucleons and mesons, built as an expansion in small momentum transfer (and meson masses) compared with the typical mass of the nucleons and the chiral symmetry breaking scale of pions interacting in loops, $\Lambda_{\chi}=4 \pi f_{\pi} \simeq 1.2 \mathrm{GeV}$, where $f_{\pi}$ is the pion decay constant.

When dealing with baryons in an effective Lagrangian context, the main difficulty is that the nucleon four momentum is of the same order as the expansion scale, since its mass is $m_{B} \simeq 1 \mathrm{GeV}$ no matter how small is the momentum transfer, and even in the chiral limit [2]. HBChPT overcomes this problem by treating the baryon fields as static heavy fermions consistently with Chiral Symmetry, following the ideas of Heavy Quark Effective Theory [3]. An slightly off-shell baryon momentum can be written as:

$p^{\mu}=m_{B} v^{\mu}+k^{\mu}, \quad$ with $\quad v \cdot k \ll m_{B}$.

Then, the Lagrangian $\mathcal{L}_{v}$ is given in terms of velocity dependent baryon fields

$B_{v}(x)=\frac{1+\not x}{2} \exp \left(i m_{B} \not v v_{\mu} x^{\mu}\right) B(x)$

satisfying now a massless Dirac equation $\not \partial B_{v}=0$ and whose momenta is $k \ll m_{B}$. Lorentz invariance is ensured by integrating with a Lorentz invariant measure, i.e. $\mathcal{L}=$ $\int \frac{d v^{3}}{2 v^{0}} \mathcal{L}_{v}$. Once this is done, it is possible to find a systematic power counting in $k / \Lambda_{\chi}$,

*Talk given at the 8th International Conference on Hadron Spectroscopy, HADRON99, August 24-28, 1999, Beijing, China. Work partially supported by DGICYT under contract AEN97-1693. 
Table 1

Values of the chiral constants from a fit of HBChPT to extrapolated threshold parameters [1] and from a IAM fit to phase shifts.

\begin{tabular}{|c|c|c|c|c|c|c|c|c|c|}
\hline & $a_{1}$ & $a_{2}$ & $a_{3}$ & $a_{5}$ & $b_{1}+b_{2}$ & $b_{3}$ & $b_{6}$ & $b_{16}-b_{15}$ & $b_{19}$ \\
\hline HBChPT & -2.6 & 1.4 & -1 & 3.3 & 2.4 & -2.8 & 1.4 & 6.1 & -2.4 \\
\hline IAM & 9.1 & -8.7 & 0.75 & 27.76 & 7.07 & -9.6 & 0.056 & 68.9 & -23.13 \\
\hline
\end{tabular}

$k / m_{B}, M / \Lambda_{\chi}$ and $M / m_{B}$, where $M$ is the mass of the mesons. Generically we will denote $M$ and $k$ by $q$.

In the minimal formulation, only the fields of the pseudoscalar meson octet and the baryon octet are used to build the effective Lagrangian. In other cases, the baryon decuplet is also considered as a fundamental field of the effective Lagrangian.

With the vertices of the Effective Lagrangian of a given order, it is possible to calculate Feynman diagrams containing loops. Each loop increases the order of the diagram so that any divergence can be absorbed in the coefficients of higher order operators. It is therefore possible to obtain finite results order by order in HBChPT, but paying the price of more and more chiral parameters.

\section{2. $\pi$-N scattering in HBChPT}

Despite its difficulty, there are some one-loop $O\left(q^{3}\right)$ HBChPT calculations in the literature [4,5]. For $\pi$-N scattering only four $O\left(q^{2}\right)$ and five $O\left(q^{3}\right)$ chiral parameters are relevant. In table I we list their estimated values obtained from a fit to the nuclear $\sigma$-term, the Goldberger-Treiman discrepancy and ten extrapolated threshold parameters [⿴囗十. With this knowledge it was possible to make six predictions of threshold parameters, which is "Neither too impressive nor discouraging" \#1. Let us remark the rather slow convergence of HBChPT, "since the contributions of the first three orders are frequently comparable". Encouraged by these results and by the success of unitarization techniques in meson-meson scattering [8.9], our aim is to extend the applicability of HBChPT to higher energies implementing unitarity.

As we have seen, within the HBChPT formalism, and counting $q_{c m}$ and $M$ as $O(\epsilon)$, the $\pi$-N amplitudes are obtained as a series in the momentum transfer. Customarily $\pi$-N scattering is described in terms of partial wave amplitudes of definite isospin $I$ and angular momentum $J$, that are therefore obtained as $t \simeq t_{1}+t_{2}+t_{3}+O\left(\epsilon^{4}\right)$, where the subscript stands for the power of $\epsilon$ that each contribution carries. However, an expansion will never satisfy exactly the $\pi$-N elastic unitarity condition

$\operatorname{Im} t=q_{c m}|t|^{2}$

although HBChPT satisfies unitarity perturbatively. Indeed, we have checked that

$\operatorname{Im} t_{3}=q_{c m}\left|t_{1}\right|^{2}$

Unfortunately, unitarity is a very relevant feature of strong interactions and it is fundamental in order to incorporate resonances and their associated poles, which cannot be 
accommodated in an energy expansion. Note that although there are other $O\left(q^{3}\right)$ calculations with an explicit $\Delta(1232)$ which reproduce phase shifts up to $E_{c m} \simeq 100 \mathrm{MeV}$ [6], our aim is the unitarization without including explicit resonance fields.

\section{The Inverse Amplitude Method and $\pi-\mathrm{N}$ scattering.}

Dividing Eq.(3) by $|t|^{2}$, the elastic unitarity condition can be recast as

$$
\operatorname{Im}(1 / t)=-q_{c m} \Longrightarrow t \simeq \frac{1}{\operatorname{Re}(1 / t)-i q_{c m}}
$$

Any amplitude in this form satisfies elastic unitarity exactly. Depending on how well we approximate the real part of the Inverse Amplitude, we have different unitarization methods. For instance, setting $\operatorname{Re}(1 / t)=\left|q_{c m}\right| \cot \delta=-\frac{1}{a}+\frac{r_{0}}{2} q_{c m}^{2}$ we have the effective range approximation. If we simply take $\operatorname{Re} t \simeq t_{1}$ we obtain a Lippmann-Schwinger type equation. Finally, if we use the $O\left(q^{3}\right)$ HBChPT expansion we arrive at:

$t \simeq \frac{t_{1}^{2}}{t_{1}-t_{2}+t_{2}^{2} / t_{1}-\operatorname{Re} t_{3}-i q_{c m} t_{1}^{2}}$

which is the $O\left(q^{3}\right)$ form of the Inverse Amplitude Method (IAM). Note that if we expand again in terms of $q$, we recover at low energies the HBChPT result.

Unitarization methods are not foreign to effective theories. Incidentally, eq.(6) is nothing but a Padé approximant of the $O\left(q^{3}\right)$ series, and it is well known that Padés, together with very simple phenomenological models are enough to describe the main features of $\pi$-N scattering. Although a systematic application with an effective Lagrangian was demanded, it was never carried out (see [7] and references therein). In recent years, with the advent of Chiral Perturbation Theory (ChPT), the IAM has been applied to mesonmeson scattering with a remarkable success [8]. In particular,and using the $O\left(p^{4}\right) \mathrm{ChPT}$ Lagrangians, the IAM reproduces all the channels up to about $1.2 \mathrm{GeV}$, including the $\sigma$, $f_{0}, a_{0}, \rho, \kappa, K^{*}$ and octet $\phi$ resonances [9]. Very recently, the Lippmann-Schwinger type equation mentioned above has been applied to S-wave kaon-nucleon scattering with eight coupled channels using the lowest order Lagrangian plus one parameter [10], reproducing all the low energy cross-sections as well as the $\Lambda(1405)$ resonance.

\section{Results and Summary}

In Fig. 1 we show the preliminary results of an IAM fit to low energy $\pi$-N scattering phase shifts. It can be noticed that there is a general improvement and we obtain a fairly good description up to at least the first inelastic threshold for channels even up to $320 \mathrm{MeV}$ of $\mathrm{CM}$ energy. Note that the $\Delta(1232)$ resonance in the $P_{33}$ channel, has been generated dynamically. Indeed we have found its associated pole in the $2^{\text {nd }}$ Riemann sheet, at $\sqrt{s}=1209-i 46 \mathrm{MeV}$.

The chiral parameters resulting from the IAM fit are given in table I. Note how different they are from those obtained in [4]. That can be due to several reasons: a) The slow convergence of the series. Indeed we have checked that contributions from different orders are comparable in almost every partial wave at the energies we use. The effect of higher order terms, which was less relevant at threshold, is absorbed in our case in the fit of the 

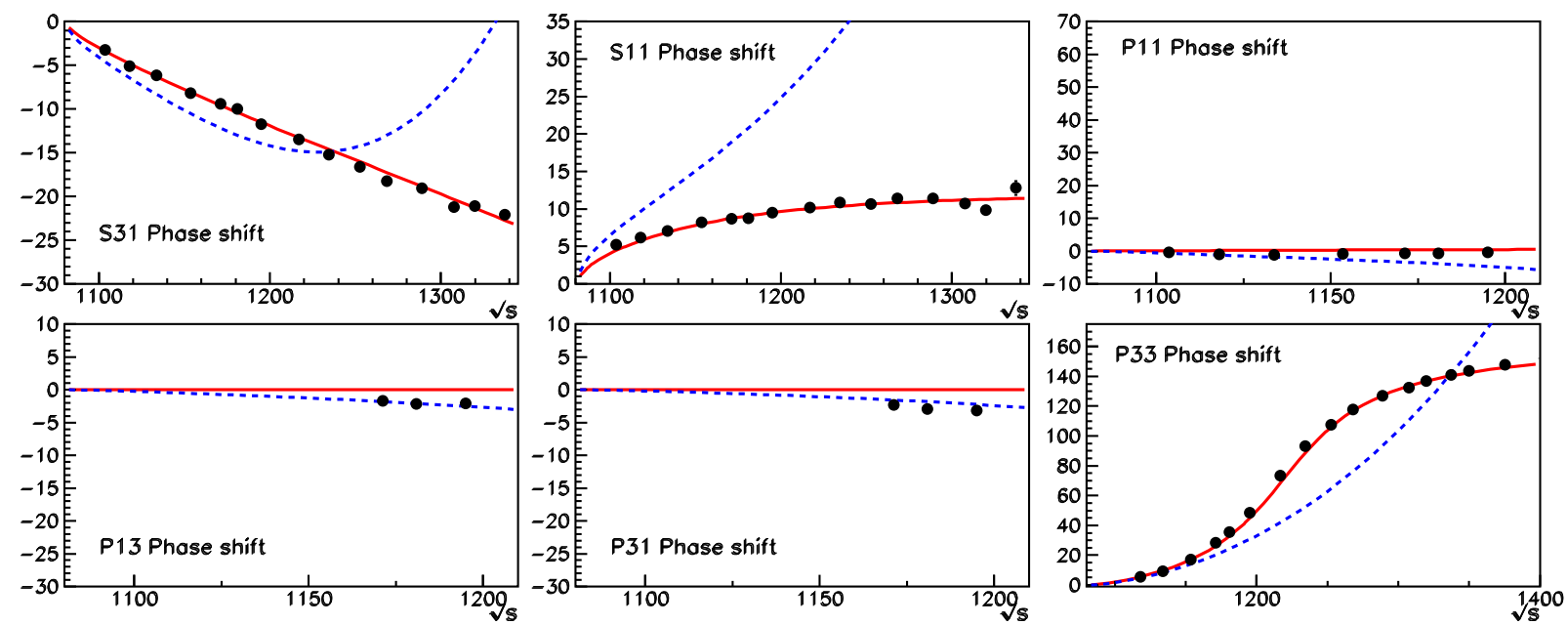

Figure 1. Phase shifts of $\pi$-N scattering. The data comes from 11 . The continuous line is the IAM fit and the dashed line is the result of HBChPT using the chiral parameters of [1].

parameters. b) Even without unitarization, the values of the parameters have a strong dependence on the observables or the range of energies used to extract them [12]. c) It could also suggest that the $\Delta$ should be included as an explicit degree of freedom in the Lagrangian. d) Also in [12] it is suggested that the data in [11 yields a too large $\sigma$ term when analyzed with HBChPT.

Further work along these lines is still in progress and a more detailed presentation with additional and more complete results will be presented elsewhere.

\section{REFERENCES}

1. E. Jenkins and A. V. Manohar, Phys. Lett. B255 (1991) 558; V. Bernard et al, Nucl. Phys. B388 (1992) 315. G. Ecker, Czech. J. Phys. 44 (1994) 405.

2. J. Gasser, M. E. Sainio and A. Svarc, Nucl. Phys. B307 (1988) 779.

3. H. Georgi, Phys. Lett. B240 (1990) 447.

4. M. Mojzis, Eur. Phys J. C 2 (1998) 181.

5. V. Bernard, N. Kaiser and U. -G. Meißner, Nucl. Phys. A615 (1997) 483; N.Fettes, U.-G. Meißner and S. Steininger, Nucl. Phys. A (1998) 199.

6. A. Datta and S. Pakvasa, Phys. Rev. D56 (1997) 4322; P.J. Ellis and H.-B. Tang, Phys. Rev. C57 (1998) 3356.

7. J.L. Basdevant, Fort. der Phys. 20 (1972) 283.

8. T. N. Truong, Phys. Rev. Lett. 661 (1988) 2526; Phys. Rev. Lett. 67 (1991) 2260; A. Dobado, M.J.Herrero and T.N. Truong, Phys. Lett. B235 (1990) 134 ; A. Dobado and J.R. Peláez,Phys. Rev. D47 4883 (1993); Phys. Rev. D56 (1997) 3057.

9. J.A. Oller, E. Oset and J.R. Peláez, Phys. Rev. Lett. 80 (1998) 3452 ; Phys. Rev. D59 (1999) 074001; and hep-ph/9909556 in this proceedings. F. Guerrero and J. A. Oller, Nucl. Phys. B537 (1999) 459.

10. E. Oset and A. Ramos, Nucl.Phys. A635 (1998) 99.

11. R. Arndt et al. nucl-th/9807087. SAID online-program.(Virginia Tech Partial-Wave Analysis Facility). Solution SP99, http://said.phys.vt.edu

12. P. Büttiker and U.-G. Meißner, hep-ph/9908247. 